\title{
IMPACT OF LEAM AND CBV CONDITIONING ON GASTROINTESTINAL TOXICITY AT EARLY PERIODS FOLLOWING HEMATOPOIETIC CELL TRANSPLANTATION: A RETROSPECTIVE STUDY
}

\author{
Cristina Paula Novaes ${ }^{1}$, DDS MD, Abrahão Elias Hallack Neto², MD PhD, Kelli Borges dos Santos ${ }^{3}$, RN PhD, Luci- \\ ana Corrêa ${ }^{4}$, DDS PhD \\ 1 Department of General Pathology, University of São Paulo, School of Dentistry. \\ 2 Hematology and Bone Marrow Transplantation Center of University Hospital, Federal University of Juiz de Fora. \\ 3 Hematology and Bone Marrow Transplantation Center of University Hospital, Federal University of Juiz de Fora. \\ 4 Department of General Pathology, University of São Paulo, School of Dentistry.
}

Running title: GASTROINTESTINAL TOXICITY AFTER LEAM AND CBV

Correspondence to: Luciana Corrêa (Icorrea@usp.br)

\begin{abstract}
Objectives: To compare the severity of oral mucositis and the frequency of gastrointestinal mucositis, and to observe if there is impact of these adverse effects on overall survival (OS), in patients who underwent CBV (carmustine, BCNU, and VP-16) and LEAM (lomustine, etoposide, Ara-C, and melphalan) conditioning for autologous hematopoietic cell transplantation (aHCT). Method: We collected retrospective data from medical records $(n=120)$ of transplantation and mucositis in the digestive tract of Hodgkin's and non-Hodgkin's lymphoma patients. Results: The frequency of OM grade 1 was higher in LEAM (36.76\%) than in CBV $(19.72 \%, p=0.038)$. There were no significant differences between the frequency of gastrointestinal mucositis in the two regimens (CBV $-52.11 \%$ and LEAM $-63.27 \%, p=0.305$ ). CBV regimen exhibited lower 1 -year overall survival $(O S)$ than did LEAM $(p=0.003)$. Oral mucositis grade $\geq 2$ was associated with reduced OS in the CBV group ( $p=0.013)$. CBV regimen ( $H R=2.98, p 0.005)$ and oral mucositis grade $\geq 2(H R=2.17, p=0.013)$ interfered negatively on the OS rate. Conclusion: Oral mucositis was more severe in CBV than in LEAM, decreasing the OS rate. Further studies with comprehensive follow-up and toxicity analyses must be undertaken to clarify the safety of LEAM conditioning in the digestive tract.
\end{abstract}

Keywords: Lymphoma. LEAM. CBV. Oral mucositis. Autologous hematopoietic cell transplantation.

\section{INTRODUCTION}

Autologous hematopoietic cell transplantation (aHCT) has been indicated for patients with refractory or relapsed Hodgkin's disease and non-Hodgkin's lymphoma, improving event-free and progression-free survival rates..$^{1-3}$ To achieve favorable outcomes, aHCT conditionings must have anti-lymphoma effects.2. High-dose chemotherapy combining cyclophosphamide, BCNU/carmustine, and VP-16/etoposide (CBV); BCNU/carmustine, etoposide, Ara-C/cytarabine, and melphalan (BEAM); or cyclophosphamide, etoposide, Ara-C/cytarabine, and carmustine (BEAC) are examples of conditionings adopted to avoid high-dose irradiation; ${ }^{2,4}$ these regimens are selected based on institutional experiences and preferences. An optimal conditioning regimen for aHCT, however, remains a prominent challenge in lymphoma treatment. ${ }^{5}$

Carmustine leads to high lung toxicity, has a high cost, and is in shortage in many countries, ${ }^{6}$ including Brazil. ${ }^{7}$ This drug has been replaced by lomustine combined with etoposide, Ara-C/cytarabine, and melphalan (LEAM) ${ }^{6-9}$ to substitute CBV in aHCT 
conditioning. Although promising results are linked to LEAM, including improvement in overall and disease-free survivals ${ }^{7}$, its toxicity in the gastrointestinal tract relative to CBV has been poorly investigated. In our institution, LEAM was introduced in 2011 to replace CBV conditioning due to carmustine shortage in the national market. However, we did not know the LEAM toxicity in the gastrointestinal tract of our patients and whether this toxicity would have an impact on overall survival. Therefore, the aim of this study was to compare the severity of oral and gastrointestinal mucositis in lymphoma patients who underwent CBV and LEAM conditionings prior to aHCT, as well as the impact of these toxicities on overall survival (OS).

\section{METHOD}

This retrospective study enrolled consecutive Hodgkin's and non-Hodgkin's lymphoma patients who underwent aHCT. The study was approved by the ethics committee of our institution (Project \#139420-2011, CAAE 0102.0.420.000-11) and followed according to the criteria defined by the Declaration of Helsinki. Informed consent from all adult patients and guardians of underage patients was obtained for aHCT-related procedures.

\section{Patient and transplantation characteristics}

We examined the medical records from patients who underwent aHCT at the Bone Marrow Transplantation Center of Hospital of Juiz de Fora University, Brazil, between September 2004 and July 2016. Inclusion criteria were: patients diagnosed with Hodgkin's and non-Hodgkin's lymphoma who underwent a conditioning regime with CBV or LEAM, who followed the oral care protocols, and whose records had clear information on oral and gastrointestinal mucositis. Exclusion criteria were medical records with insufficient information about time duration of neutropenia and transplantation, and about oral and gastrointestinal toxicity. Medical records of patients who refused the oral care protocols were also excluded.

\section{Data collection}

The data were collected by a single researcher with expertise in bone marrow transplantation. The following information about the patient and the transplantation was collected: age, sex, primary disease, status of the disease (refractory, partial, or complete remission), duration of neutropenia (number of days with neutrophil count $\leq 500$ cells/mm3), and duration of hospitalization (number of days from the first day of conditioning to the day of discharge from the transplantation center). Data on oral mucositis and diarrhea were collected from the first day of the conditioning to the last day in the bone marrow transplantation. Presence of diarrhea related to gastrointestinal mucositis was considered only when non-infectious etiology (confirmed by microbial cultures) and $\geq 3$ daily episodes were registered. We also recorded the presence of prescriptions for artificial nutrition.

\section{LEAM and CBV conditionings}

Patients who underwent HCT from 2004 to 2011 received the CBV conditioning; from 2011 to 2016, all the selected patients were exposed to the LEAM conditioning. Patients in the LEAM group received lomustine $(300 \mathrm{mg} / \mathrm{m} 2)$ in $\mathrm{D}-4$, etoposide $(1000$ $\mathrm{mg} / \mathrm{m} 2)$ in $\mathrm{D}-3$, aracytin $(4000 \mathrm{mg} / \mathrm{m} 2)$ in $\mathrm{D}-2$, and melphalan $(140 \mathrm{mg} / \mathrm{m} 2)$ in D-1. Dosages for LEAM conditioning regimen were according to Dos Santos et al. (9). Hematopoietic cells were infused $24 \mathrm{~h}$ following the end of the melphalan conditioning. In CBV group, the patients received cyclophosphamide $(1800 \mathrm{mg} / \mathrm{m} 2)$ from D-6 to D-3 (total of $7200 \mathrm{mg} /$ $\mathrm{m} 2)$, etoposide $(400 \mathrm{mg} / \mathrm{m} 2)$ every $12 \mathrm{~h}$ from D-6 to D-4 (total of $2400 \mathrm{mg} / \mathrm{m} 2)$, and BCNU $(450 \mathrm{mg} / \mathrm{m} 2)$ in $\mathrm{D}-2$.

\section{Oral care and oral mucositis assessment}

All patients received oral hygiene guidance and an oral care protocol for prevention and treatment of oral mucositis. The patients used a soft toothbrush and toothpaste with fluoride, and alcohol-free antiseptic mouthwash for $30 \mathrm{~s}$ twice per day. Low-level laser therapy was administered three times per week using a diode laser (gallium indium arsenide, InGaA$\mathrm{IP}, 660 \mathrm{~nm}, 0.04 \mathrm{~cm} 2 \mathrm{spot}, 100 \mathrm{~mW}, 25 \mathrm{~J} / \mathrm{cm} 2,10 \mathrm{~s}$ per point, $1 \mathrm{~J}$ per point) from the first day of oral mucositis symptoms to the point of complete remission of the lesions.

Oral mucositis severity was recorded daily by a dentist following the World Health Organization grading criteria as follows: 0 - absence of oral lesions; 1 - only erythema; 2 - presence of pseudomembrane or ulceration, but normal oral ingestion is possible; 3 presence of ulcerated lesions, and only liquid diet is possible by oral ingestion; 4 - presence of ulcerated lesions, and oral ingestion is not possible; necessary artificial nutrition. 


\section{Primary and secondary outcomes}

The primary outcome was the impact of CBV and LEAM regimens on the severity of oral and gastrointestinal mucositis. The secondary outcome was the impact of oral and gastrointestinal mucositis on overall survival (OS).

\section{Statistical analyses}

Numerical and categorical data were shown as median and minimum-maximum, and absolute and relative (\%) frequencies, respectively. The medical records were grouped in LEAM and CBV conditionings. Comparisons between the two groups were performed using the Mann-Whitney test and $x 2$ test with Bonferroni correction. In each group, we considered duration of neutropenia and transplantation (dichotomized in accordance with the median of days), presence of non-Hodgkin's lymphoma, and disease status (in partial remission/refractory status) as risk factors for oral mucositis grade $\geq 2$ and for presence of gastrointestinal mucositis. The Kaplan-Meier curve was used to measure OS, which was defined as the first day of enrollment in the hospital to the last day of the follow-up registration. Mean follow-up was one and three years in the LEAM and CBV conditioning groups, respectively. We applied log-rank test to compare the OS between the two groups and to verify the impact of oral mucositis and diarrhea in the OS. Cox proportional hazards regression was applied to determine which factor was decisive for OS. We adopted $5 \%$ as the level of statistical significance.

\section{RESULTS}

From September 2004 to July 2016, a total of 286 aHCTs were performed in our institution. Of these, 128 were performed on patients with a diagnosis of either Hodgkin's or non-Hodgkin's lymphoma. Eight medical records showed inconsistent data, leading to a total of 120 medical records selected for the study.

\section{Patients and transplantation characteristics}

Table 1 depicts patient and transplantation characteristics. The CBV and LEAM groups were composed by 71 and 49 patients, respectively. The median age was 34 years, and the majority were male (64.17\%). For both groups, the most frequent primary disease was Hodgkin's lymphoma, nodular sclerosis subtype. In the LEAM group, the frequency of patients with complete remission of the disease (51.0\%) was higher than in the CBV group (32.4\%, $p=0.009$ ). In addition, the median duration of neutropenia in LEAM
(8.5 days) was significantly lower than in CBV (12 days, $p<0.001$ ); a similar result was found for length of hospitalization (LEAM - 18 days vs. CBV - 21 days, $p=0.014$ ).

\section{Oral and gastrointestinal mucositis}

Table 2 shows the data of oral and gastrointestinal mucositis, as well as of artificial nutrition prescription. Oral mucositis was detected in $52.5 \%$ of patients. The frequency of OM grade 1 was higher in LEAM (36.7\%) than in CBV (19.7\%, p = 0.038). Grade 4 oral mucositis was not observed in any group. No significant differences were found in the frequency of gastrointestinal mucositis between the two regimens (CBV - 52.1\%, LEAM - 63.3\%). The percentage of artificial nutrition prescription was very low in both groups (CBV $-2.8 \%$, LEAM $-2.0 \%$ ). Analyzing the potential risk factors for oral mucositis grade $\geq 2$ and gastrointestinal mucositis (Table 3), no significant association was found in either group for any variables.

\section{Overall survival}

The CBV regimen resulted in lower 1-year OS (mean: $64.0 \%, 95 \%$ Cl: $51.4-74.0 \%$ ) than did the LEAM regimen (mean: $80.0 \%, 95 \% \mathrm{Cl}: 64.0-89.0 \%, \mathrm{p}=0.003$; Fig. 1). Oral mucositis grade $\geq 2$ significantly reduced the OS in the CBV group ( $p=0.013$; Fig. $2 A)$, but not in the LEAM group (Figure 2B). In the CBV group, OS in patients with gastrointestinal mucositis was lower than the OS of the CBV patients without gastrointestinal mucositis ( $p=0.050$; Fig. $2 C$ ); this trend was not observed in the LEAM group ( $p=0.740$; Fig. $2 D$ ). Duration of neutropenia and transplantation, primary disease, status of the disease, previous radiotherapy, and number of previous chemotherapies did not significantly influence $O S$.

We performed a Cox regression to verify whether the presence of gastrointestinal mucositis, oral mucositis grade $\geq 2$, or conditioning with CBV affected OS. In the second model, after omitting gastrointestinal mucositis (Table 4), oral mucositis grade $\geq 2$ and CBV regimen were found to significantly impact OS.

\section{DISCUSSION}

In this retrospective study, our goal was to compare oral and gastrointestinal mucositis between patients having undergone CBV and LEAM regimens. We found that the frequency of oral mucositis with mild severity was significantly higher in the LEAM group than in the CBV group. In addition, oral mucositis grade $\geq 2$ reduced the OS rate in the CBV group, 
suggesting that in CBV, the oral toxicity had a high impact.

The conditioning type was the only predictive factor for mucositis in the digestive tract in the present study. None of other factors related to the patient and the transplantation were linked to mucositis. It is important to mention that we failed to find other studies (apart from those of our group) analyzing the severity of mucositis in LEAM and CBV conditionings.

On the other hand, there are studies comparing LEAM with BEAM conditioning, which showed a lower frequency of severe oral mucositis in LEAM; however, these differences were not statistically significant. $6,8,10$ Other investigations reported similar trends in oral toxicity between these two regimens. ${ }^{11}$ Comparing BEAM and CBV, BEAM toxicity appears to be controversial. For instance, one study showed that the frequency of oral mucositis and diarrhea was higher in BEAM than in CBV,4 but another report found the opposite. ${ }^{12}$

Although we found differences in oral mucositis, this trend was not detected in gastrointestinal mucositis. The present study neglected the impact of gastrointestinal mucositis, addressing only its frequency, and not its severity. Other authors reported a lower rate of gastrointestinal toxicity in LEAM relative to BEAM. ${ }^{6,10}$

Besides high oral toxicity, the CBV regimen also presented longer durations of neutropenia compared to the LEAM regimen. Another study showed that CBV resulted in a longer duration of neutrophil engraftment than did BEAM. ${ }^{12}$ We also detected that the length of hospitalization was reduced in the LEAM group, with a median of 18 days; in the literature, the hospitalization period of patients who underwent LEAM conditioning was $>20$ days. ${ }^{6,10,11}$

Despite the differences between the two conditionings, the oral mucositis was, in general, not severe in the majority of the patients, and the prescription of artificial nutrition was rare. This trend is in accordance with the other study that investigated LEAM and BEAM. ${ }^{11}$ The routine oral care protocol may have contributed to this lower toxicity.

Survival in LEAM regimens has been considered similar to that observed in BEAM regimens.6,8,10 However, the follow-up period in these investigations is quite short, which limits the veracity and generalizability of any conclusions drawn. We found a significant improvement in OS in patients who underwent LEAM relative to CBV, but our follow-up period (only one year) was also substantially limited. A previous study from our group reported better OS in LEAM than in CBV, but the analysis was restricted to 100 days post-transplantation.? It is important to mention that some patients who were retrospectively included in the present study were analyzed prospectively in our prior study, which restricts any comparisons made between the two studies.

One of the main findings was that, in addition to CBV conditioning, oral mucositis grade $\geq 2$ impacted negatively on OS, suggesting the importance of oral mucositis in relation to other risk factors in the transplantation. Therefore, the oral care protocols to prevent and treat oral mucositis should be emphasized in $\mathrm{CBV}$ regimens.

\section{CONCLUSION}

In conclusion, the severity of oral mucositis is higher in CBV than in LEAM, impacting negatively on the OS rate. Further studies, with comprehensive follow-up and toxicity analyses must be conducted to clarify the safety of LEAM conditioning for the digestive tract.

Acknowledgments: We thank all the staff of Bone Marrow Transplantation Unit at University Hospital of Federal University of Juiz de Fora, for the clinical support and medical records retrieval. 


\section{REFERENCES}

1. Perales MA, Ceberio I, Armand P, Burns LJ, Chen $\mathrm{R}$, Cole PD, et al. Role of cytotoxic therapy with hematopoietic cell transplantation in the treatment of Hodgkin lymphoma: guidelines from the American Society for Blood and Marrow Transplantation. Biol Blood Marrow Transplant. 2015;21(6):971-83.

2. Lukenbill J, Hill B. Relapsed/refractory diffuse large B-cell lymphoma: review of the management of transplant-eligible patients. Leuk Lymphoma. 2015;56(2):293-300.

3. Kharfan-Dabaja MA, Kumar A, Ayala E, Hamadani $M$, Reimer P, Gisselbrecht $C$, et al. Clinical Practice Recommendations on Indication and Timing of Hematopoietic Cell Transplantation in Mature T Cell and NK/T Cell Lymphomas: An International Collaborative Effort on Behalf of the Guidelines Committee of the American Society for Blood and Marrow Transplantation. Biol Blood Marrow Transplant. 2017;23(11):1826-38.

4. Shi Y, Liu P, Zhou S, Yang J, Han X, He X, et al. Comparison of CBV, BEAM and BEAC high-dose chemotherapy followed by autologous hematopoietic stem cell transplantation in non-Hodgkin lymphoma: Efficacy and toxicity. Asia Pac J Clin Oncol. 2017;13(5):423-9.

5 Damaj G, Cornillon J, Bouabdallah K, Gressin R, Vigouroux S, Gastinne T, et al. Carmustine replacement in intensive chemotherapy preceding reinjection of autologous HSCs in Hodgkin and non-Hodgkin lymphoma: a review. Bone Marrow Transplant. 2017;52(7):941-9.

6. Sharma A, Kayal S, lqbal S, Malik PS, Raina V. Comparison of BEAM vs. LEAM regimen in autologous transplant for lymphoma at AlIMS. Springerplus. 2013;2:489.
7. Santos KB, Costa LJ, Bettarello G, Abreu MM, Mayrink GT, Mota MA, et al. LEAM versus CBV for conditioning in autologous hematopoietic stem cell transplantation for lymphoma. Bone Marrow Transplant. 2019;54(4):625-8.

8. Ramzi M, Mohamadian M, Vojdani R, Dehghani $M$, Nourani $H$, Zakerinia $M$, et al. Autologous noncryopreserved hematopoietic stem cell transplant with CEAM as a modified conditioning regimen in patients with Hodgkin lymphoma: a single-center experience with a new protocol. Exp Clin Transplant. 2012;10(2):163-7.

9. Santos KB, Costa LJ, Atalla A, Pereira J, Hallack $A E$ Neto. Lomustine use in combination with etoposide, cytarabine and melphalan in a brief conditioning regimen for auto-HSCT in patients with lymphoma: the optimal dose. Bone Marrow Transplant. 2014;49(9):1239-40.

10. Colita A, Colita A, Bumbea H, Croitoru A, Orban C, Lipan LE, et al. LEAM vs. BEAM vs. CLV Conditioning Regimen for Autologous Stem Cell Transplantation in Malignant Lymphomas. Retrospective Comparison of Toxicity and Efficacy on 222 Patients in the First 100 Days After Transplant, On Behalf of the Romanian Society for Bone Marrow Transplantation. Front Oncol. 2019;9:892.

11. Kothari J, Foley M, Peggs KS, Mackenzie S, Thomson K, Morris E, et al. A retrospective comparison of toxicity and initial efficacy of two autologous stem cell transplant conditioning regimens for relapsed lymphoma: LEAM and BEAM. Bone Marrow Transplant. 2016;51(10):1397-9.

12. Puig N, de Rubia J, Remigia MJ, Jarque I, Martín G, Cupelli L, et al. Morbidity and transplant-related mortality of CBV and BEAM preparative regimens for patients with lymphoid malignancies undergoing autologous stem-cell transplantation. Leuk Lymphoma. 2006;47(8):1488-94. 
TABLE 1 - Characteristics of the patients and the transplantation in CBV and LEAM groups.

\begin{tabular}{|c|c|c|c|c|c|c|c|}
\hline \multirow{2}{*}{ Sex $-\mathrm{n}(\%)$} & \multicolumn{2}{|c|}{$\begin{array}{c}\text { CBV } \\
(n=71)\end{array}$} & \multicolumn{2}{|c|}{$\begin{array}{l}\text { LEAM } \\
(\mathrm{n}=49)\end{array}$} & \multirow[t]{2}{*}{ P value* } & \multicolumn{2}{|c|}{ All $(n=120)$} \\
\hline & & & & & & & \\
\hline Male & 44 & $(61.97)$ & 33 & $(67.35)$ & \multirow{2}{*}{0.546} & 77 & $(64.2)$ \\
\hline Female & 27 & $(38.03)$ & 16 & $(32.65)$ & & 43 & $(35.8)$ \\
\hline Age (y) - median (range) & 33 & $(8-68)$ & 37 & $(14-67)$ & 0.088 & 34 & $(8-68)$ \\
\hline \multicolumn{8}{|l|}{ Primary disease - $\mathrm{n}(\%)$} \\
\hline Non-Hodgkin lymphomas & 24 & $(33.8)$ & 23 & $(46.9)$ & 0.208 & 47 & (39.2) \\
\hline Diffuse large B-cell & 13 & $(18.3)$ & 6 & $(12.2)$ & & & \\
\hline High-grade B-cell & 0 & $(0.0)$ & 2 & $(4.1)$ & & & \\
\hline Follicle center B-cell & 0 & $(0.0)$ & 1 & $(2.0)$ & & & \\
\hline Burkitt & 1 & $(1.4)$ & 0 & $(0.0)$ & & & \\
\hline Mantle cell & 4 & (5.6) & 8 & $(16.3)$ & & & \\
\hline Peripheral T-cell & 3 & $(4.2)$ & 4 & $(8.2)$ & & & \\
\hline Anaplastic large cell & 1 & (1.4) & 2 & $(4.1)$ & & & \\
\hline No information & 2 & (2.8) & 0 & $(0.0)$ & & & \\
\hline Classical Hodgkin lymphomas & 47 & $(66.2)$ & 26 & $(53.1)$ & & 73 & $(60.8)$ \\
\hline Nodular sclerosis & 32 & $(45.1)$ & 20 & $(40.8)$ & & & \\
\hline Mixed cellularity & 10 & $(14.1)$ & 4 & $(8.2)$ & & & \\
\hline Lymphocyte-rich & 2 & $(2.8)$ & 2 & $(4.1)$ & & & \\
\hline No information & 3 & $(4.2)$ & 0 & $(0.0)$ & & & \\
\hline Previous radiotherapy $-\mathrm{n}(\%)$ & 36 & $(50.7)$ & 17 & $(34.7)$ & 0.211 & 53 & (44.6) \\
\hline $\begin{array}{l}\text { Number of previous chemotherapy - median } \\
\text { (range) }\end{array}$ & 2 & $(1-5)$ & 2 & $(1-4)$ & $<0.001$ & 2 & $(1-5)$ \\
\hline \multicolumn{8}{|l|}{ Disease status - $\mathrm{n}(\%)$} \\
\hline Partial remission & 34 & $(47.9)$ & 17 & $(34.7)$ & 0.754 & 51 & $(42.5)$ \\
\hline Complete remission & 23 & $(32.4)$ & 25 & $(51.0)$ & 0.009 & 48 & $(40.0)$ \\
\hline Refractory & 9 & $(12.7)$ & 5 & $(10.2)$ & 0.678 & 14 & $(11.6)$ \\
\hline Without data & 5 & $(7.0)$ & 2 & $(4.0)$ & 0.496 & 7 & $(5.8)$ \\
\hline Days of neutropenia + - median (range) & 12 & $(8-26)$ & 8.5 & $(5-18)$ & $<0.001$ & 10 & $(5-26)$ \\
\hline Days of hospitalization $\S-$ median (range) & 21 & $(11-74)$ & 18 & $(7-70)$ & 0.014 & 20 & $(7-79)$ \\
\hline
\end{tabular}

CBV - cyclophosphamide, BCNU, and VP-16 conditioning; LEAM - lomustine, etoposide, Ara-C, and melphalan conditioning.

* $p$ value for $c 2$ test and Mann-Whitney test.

$+<500$ cells $/ \mathrm{mm} 3$

$\S$ From the first day of conditioning to the discharge of the transplantation center. 
TABLE 2 - Frequency of digestive tract, liver, and lung toxicities, and prescription of artificial nutrition in CBV and LEAM groups.

\begin{tabular}{|c|c|c|c|c|c|c|c|}
\hline \multirow[b]{2}{*}{$\begin{array}{c}\text { Grade } \\
+ \text { of oral mucositis }-\mathrm{n}(\%)\end{array}$} & \multicolumn{2}{|c|}{$\begin{array}{c}\text { CBV } \\
(n=71)\end{array}$} & \multicolumn{2}{|c|}{$\begin{array}{l}\text { LEAM } \\
(n=49)\end{array}$} & \multirow[t]{2}{*}{ P value* } & \multicolumn{2}{|c|}{$\begin{array}{c}\text { ALL } \\
(n=120)\end{array}$} \\
\hline & & & & & & & \\
\hline 0 & 38 & $(53.2)$ & 19 & $(38.8)$ & 0.160 & 57 & $(47.5)$ \\
\hline 1 & 14 & (19.7) & 18 & $(36.7)$ & 0.038 & 32 & $(26.7)$ \\
\hline 2 & 9 & $(12.7)$ & 8 & $(16.3)$ & 0.766 & 17 & $(14.2)$ \\
\hline 3 & 10 & $(14.0)$ & 4 & $(8.1)$ & 0.481 & 14 & $(11.7)$ \\
\hline 4 & 0 & $(0.0)$ & 0 & $(0.0)$ & 1.000 & 0 & $(0.0)$ \\
\hline Gastrointestinal mucositis - $\mathrm{n}(\%)$ & 37 & $(52.1)$ & 31 & $(63.3)$ & 0.305 & 68 & $(56.7)$ \\
\hline Veno occlusive disease - $\mathrm{n}(\%)$ & 6 & $(8.4)$ & 4 & $(8.1)$ & 1.000 & 10 & $(8.3)$ \\
\hline Lung toxicity - n (\%) & 9 & $(12.7)$ & 6 & $(12.2)$ & 1.000 & 15 & $(12.5)$ \\
\hline Artificial nutrition - $\mathrm{n}(\%)$ & 2 & $(2.8)$ & 1 & $(2.0)$ & 1.000 & 3 & $(2.5)$ \\
\hline
\end{tabular}

CBV - cyclophosphamide, BCNU, and VP-16 conditioning; LEAM - lomustine, etoposide, Ara-C, and melphalan conditioning * $p$ value for $c 2$ test.

+ In accordance of World Health Organization classification.

TABLE 3 - Univariate analysis for factors associated to oral mucositis grade $\geq 2$ and gastrointestinal mucositis in CBV and LEAM groups.

\begin{tabular}{c|c|c|c|c|c|c}
\hline & \multicolumn{4}{|c}{ P values* } \\
\hline & \multicolumn{2}{|c|}{ Oral mucositis grade $\geq 2$} & \multicolumn{3}{c}{ Gastrointestinal mucositis } \\
\hline & CBV & LEAM & All & CBV & LEAM & All \\
\hline Days of neutropenia $\geq 12$ & 0.187 & 0.488 & 0.813 & 0.438 & 1.000 & 0.556 \\
\hline Days of transplantation $\geq 21$ & 0.785 & 0.173 & 0.532 & 0.808 & 0.127 & 0.583 \\
\hline Non-Hodgkin lymphoma (yes/no) & 0.278 & 1.000 & 0.397 & 0.623 & 0.390 & 1.000 \\
\hline Previous radiotherapy (yes/no) & 0.424 & 1.000 & 1.000 & 1.000 & 0.355 & 1.000 \\
\hline Previous chemotherapies $\geq 2$ & 1.000 & 0.503 & 0.678 & 0.770 & 0.758 & 0.754 \\
\hline Partial remission/refractory (yes/no) & 0.774 & 0.083 & 0.386 & 0.605 & 0.771 & 0.444 \\
\hline
\end{tabular}

CBV - cyclophosphamide, BCNU, and VP-16 conditioning; LEAM - lomustine, etoposide, Ara-C, and melphalan conditioning

* $p$ value for $c 2$ test.

TABLE 4 - Cox proportioznal harzard regression for overall survival with oral mucositis grade $\geq 2$, gastrointestinal mucositis, and CBV conditioning as explanatory variables.

\begin{tabular}{c|c|c|c}
\hline & HR & 95\%Cl & P value \\
\hline Gastrointestinal mucositis & 1.65 & $0.89-3.07$ & 0.110 \\
\hline Oral mucositis grade $\geq 2$ & 2.17 & $1.17-4.03$ & 0.013 \\
\hline CBV conditioning & 2.98 & $1.38-6.41$ & 0.005 \\
\hline
\end{tabular}

CBV - cyclophosphamide, BCNU, and VP-16 conditioning. 
FIGURE 1 - Kaplan-Meier and log rank test for overall survival in CBV and LEAM groups.

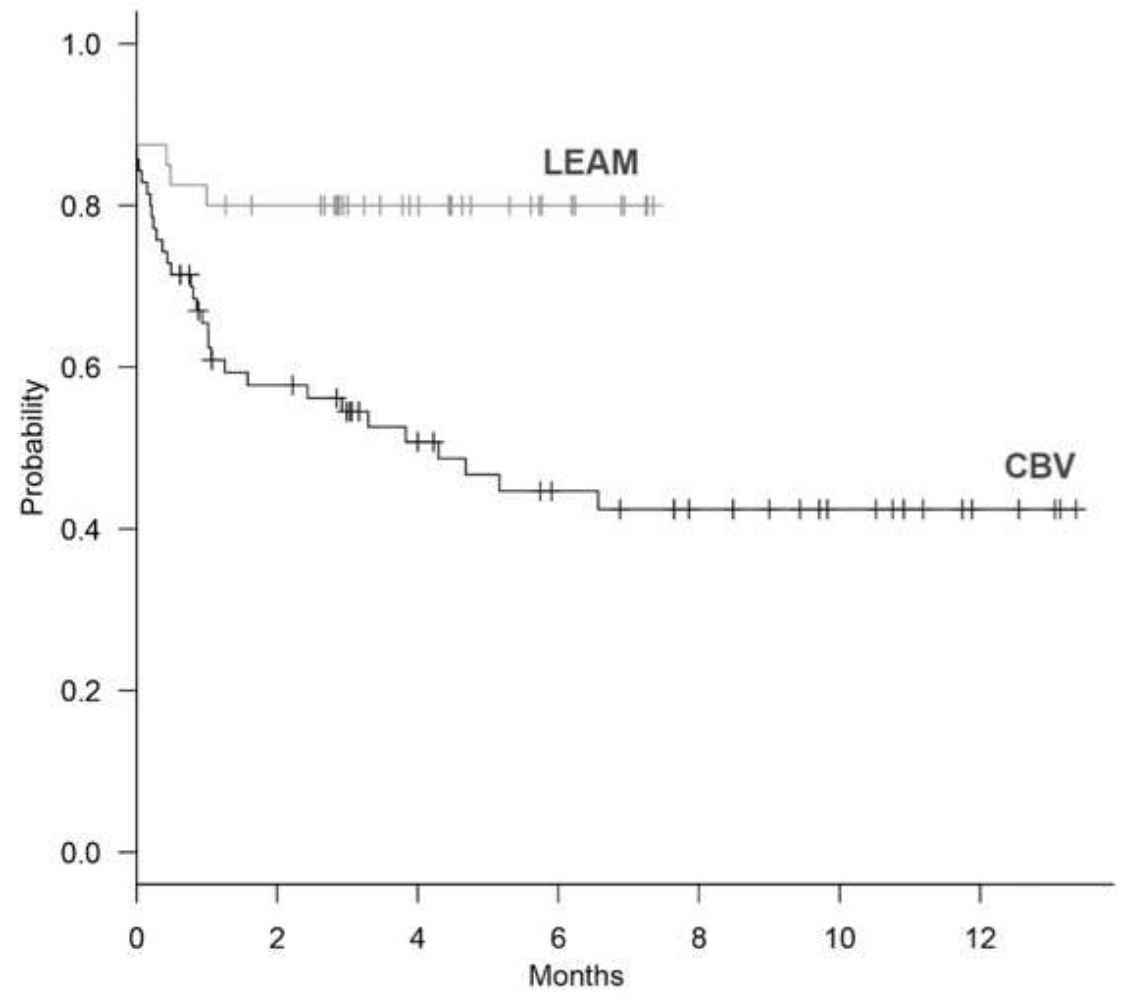

FIGURE 2 - Kaplan-Meier and log rank test for overall survival related to oral mucositis grade $\geq 2$ and gastrointestinal mucositis in CBV ( $A$ and $C$ ) and LEAM groups ( $B$ and $D)$.
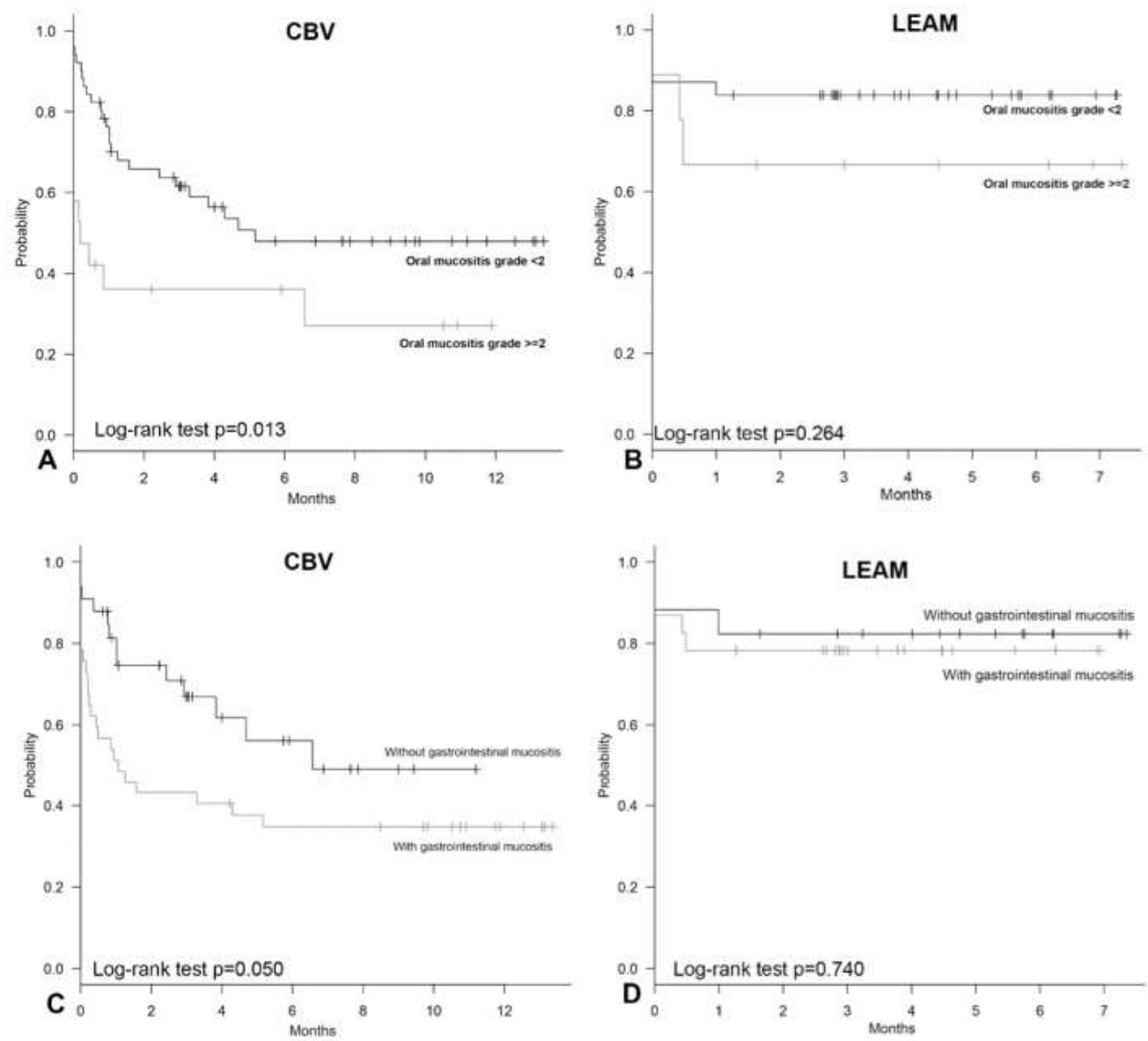